\title{
Search for variants of the gene-promoter and the potential phosphotyrosine encoding sequence of the insulin receptor substrate-2 gene: evaluation of their relation with alterations in insulin secretion and insulin sensitivity
}

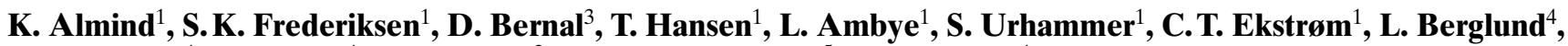 \\ R. Reneland ${ }^{4}$, H. Lithell ${ }^{4}$, M.F. White ${ }^{2}$, E. Van Obberghen ${ }^{5}$, O. Pedersen ${ }^{1}$
}

${ }^{1}$ Steno Diabetes Centre and Hagedorn Research Institute, Copenhagen, Denmark

2 Joslin Diabetes Center, Harvard Medical School, Boston, Mass., USA

${ }^{3}$ Department Biochemistry and Molecular Biology, University of Valencia, Spain

${ }^{4}$ Department of Geriatrics, Uppsala University, Sweden

${ }^{5}$ Inserm U 145, Medical Faculty, University of Nice, France

\section{Abstract}

Aims/hypothesis. The aim of this study was to screen part of the putative promoter sequence in addition to 14 potential phosphotyrosine residues of human IRS-2 for genetic variability which might cause changes in protein expression or function. Furthermore, the potential impact on insulin secretion and sensitivity of a previously identified IRS-2 variant (Gly1057Asp) was analysed

Methods. The screenings were carried out by the SSCP-heteroduplex technique on DNA from Type II (non-insulin-dependent) diabetic patients. The impact of the Gly1057Asp variant was analysed in four glucose-tolerant Scandinavian study groups.

Results. The results showed no nucleotide substitutions in the promoter sequence, however, a novel heterozygous amino acid variant was identified (Leu647Val). In an association study, the new variant was found in 3 of 413 diabetic patients and in none of 280 glucose tolerant subjects. The variant did not affect the binding of IRS-2 to the insulin receptor or p $85 \alpha$ of phosphatidylinositol 3-kinase when measured in the yeast two-hybrid system. Examination of the common Gly1057Asp variant in 363 young healthy subjects and in 228 glucose tolerant offspring of one diabetic parent showed no differences in insulin secretion or insulin sensivity after an intravenous glucose tolerance test. Glucose tolerant middle-aged subjects homozygous for the polymorphism $(n=31)$, however, had on average a $25 \%$ decrease in fasting serum insulin concentrations $(p=0.009)$ and $28 \%$ $(p=0.01)$ and $34 \%(p=0.003)$ reductions in serum insulin concentrations at 30 and $60 \mathrm{~min}$, respectively, during an OGTT compared with wildtype carriers $(n=107)$. In a cohort of 639 elderly Swedish men the amino acid variant did not have any detectable impact on insulin secretion after an OGTT.

Conclusion/interpretation. No genetic variability was found in the IRS- 2 promoter. A rare IRS-2 variant at codon 647 has been identified in Type II diabetic patients. The prevalent codon 1057 polymorphism had no consistent effect on insulin secretion or insulin sensitivity. [Diabetologia (1999) 42: 1244-1249]

Keywords Insulin receptor substrate-2, mutations, Type II diabetes, insulin secretion, insulin sensitivity.
Received: 25 January 1999 and in final revised form: 30 April 1999

Corresponding author: K. Almind, MSci. Steno Diabetes Centre, Niels Steensens Vej 2, DK-2820 Gentofte, Copenhagen, Denmark

Abbreviations: SSCP, Single strand conformation polymorphism; IGT, impaired glucose tolerance; IVGTT, intravenous glucose tolerance test; OGTT, oral glucose tolerance test; KRLB, kinase regulatory loop binding.
The importance of the insulin receptor substrate-2 (IRS-2) has recently been investigated in mice in which the homozygous deficiency of IRS-2 causes a Type II (non-insulin-dependent) diabetes-like phenotype due to both insulin resistance and pancreatic beta-cell failure [1]. The insulin resistance is profound in both skeletal muscle and liver. Morphometric analysis of the pancreas showed that the mice have considerably reduced beta-cell mass which consequently prevents an adequate insulin secretion to compensate for the insulin resistance. The study of 
Table 1. Nucleotide sequence of the putative human IRS-2 promoter 1500 basepairs upstream of the ATG start site

CTGCAGCGGTGGGGGGAGAGGACGCCCCTGCGGAAGGAAGGCGGCGCTCCTGCGGGGGCTGGAGAGACGCCCGCGCGGTCTCCCGGCTCAGGGACCGCTGGCTCGCGGGTCCGCCCTCTCCCCGGCGCTATGGAAACCGCACTTTCTCCGCCTGCCGTCCCCGATCTCTTTCTCCTATTACCTCCCAAACGCGATTTCAACTTCCCGACAAGCTGACAAGTGAATGGCGCAGAGGACGCCCCGTAAAGCGCCCGCGAGCGGGTGGGACACAGGCGCCCCCTCCGCGCCCCTCTGTCCTCCACGGAAGGGCGCGCGCCGCTCTCGATCTGGAGCGCGGTGCTCGCGAACCCGCGGCCGCGGGCCCTGGAACCTCCCCGCGCTTCGCGGCTGCGGGGGACGGAGATGCGGACGGGAGACGGGGACGGGAAACGGGGGACGGGGATGGGGCGCGCCCACCCGAGCGCGGGAGCGGCCGCCCTCGCCCAAAATGGGGACCCCGCGCCCCGGAGAGCACGGCCCGGCTCGCCGCCCATGCTTCCACTGCAAGGCCGCAGCCCCCGCCGCCGATCCCGATCCCCCGCCCCGCCCAGCCGCGGCCCCGCCCGGCGCCCCCTCCCCGGCGGAAGCTCAAGCCCAATTAATTGAGTCCGAGGCGGGAGGGAAGGGCCCTGCGCGCCGTGGCCCGCCCCGCCCCTCTTCCGCGCCCCTTTTCCCGCCCTGGGTGGCATCTCCTCCGCCGGCATCCACAACAAGCCGCTGATTAATGAGGCCGGGGCCGCCCCACCCCGCCCGGCCGGGCCGGGGCCTCCGCGGGAGGGGGGAGGGACGGCGGGAAACGCGGCCCGGGGAGAAAGGGGGGCGGGGCGGGGGCGCGCGGCCCGCCCCCGCGAGCGCCGCGCCGATTGGCCGAGCGCGCCGTCCGTCGGGGGGCGCGGCGCCAATGCGAGGCAGCGGGGCGGGGCGGCCGCGCTGTGTGTGCCTGCGTAACGCCGAGTCACATGTTGTTTTGCTCTTCTTAGTTCAGTCACTCGGTGCGCGATGTGTTTACTCACTGTGCGGCGGCGGGACCGCGACGAGCCCGGGTCGCCGTTGGCAGCAGCAGCAGCAACACCAGCAGCAGCAGCAGCCCCGGCGGCGGCGCGGACCCCGAGCGCCCGGGGCGCACCCCGGCTTCCCGGAGCGCGACGCGGCGGCAGCAGCCCCGGTGCGGCCGCGCGCGCCTTAGGCTCGGCCCCGCGGCTCGGGGACCCCGACTCCCGGCCCAGCGAGCGCGTCCCCCGGCGCCGCCCGAGAGCCCGAGGAGGCAGCGGCCGCAGGCAGCCGGGGAGGGGGGCGGCCACCGCCCGCGCCGGGCATCCTCAGGAGCCCCAGAGCGCGGAGGGCGCGGCGCCGCCGAGCGGTGCTGGCCCCCGCGGGCCTCCCCGGACCTTCCCCACCGCCTGGGCCCGAGGGACGCGTGATCGGGCGGGCGGCCGGGCGCAAGGGTGGGAGGGAGCCGCCCCCGCCCGCGCCCCCTCCGCCCCTCGCCCCAACCCCTGGGCGCCGGGCCCGGGCCGCGCGGCCTGAAGCGCCCGCGATG

the IRS-2 deficient mice therefore suggests that IRS2 plays a unique part in the regulation of beta-cell neogenesis, proliferation and survival. We have previously studied the genetic variability of the coding region of human IRS-2 [2] and reported on two nucleotide substitutions that predicted amino acid changes. One of these was a rare variant at codon 879 which resulted in a glycine to a serine substitution; the other was at codon 1057, had an allelic frequency of $34 \%$ in both control subjects and Type II diabetic patients and predicted a replacement of glycine by aspartic acid. A possible effect of the latter amino acid substitution for the function of IRS-2 is difficult to predict from the primary structure of the protein. We failed to show an association of the codon 1057 polymorphism with an increased prevalence of Type II diabetes [2]. The purpose of the present study, therefore, was to elucidate whether the prevalent polymorphism at codon 1057 in human IRS-2 was associated with intermediary pre-diabetic phenotypes by studying insulin secretion and insulin sensitivity in glucose tolerant subjects from four Scandinavian population samples. In addition, we scanned by the SSCP-heteroduplex technique the human IRS-2 promoter for nucleotide changes which potentially could have an impact on the expression of IRS-2 and furthermore, extended our previous mutation analysis of the coding region by scanning five DNA segments comprising a total of 14 tyrosine residues on DNA from 318 Type II diabetic patients. Tyrosine residues were chosen for a selective mutation search, since we hypothesized that genetic variability in or close to these potential recognition motifs for signaling proteins might cause altered IRS-2 function.

\section{Subjects and methods}

Study groups. Phenotype studies were done in three Danish and one Swedish Caucasian study groups. One Danish group consisted of 363 young healthy subjects aged 18-32 years who in 1979 and again in 1984 and 1985 as children had participated in blood pressure surveys in a representative and specified part of Copenhagen [3]. These subjects had been examined by an IVGTT in combination with intravenous tolbutamide injection [3]. The other Danish group consisted of 228 glucose tolerant offspring of one Type II diabetic parent. These subjects were examined by an IVGTT in combination with intravenous injection of tolbutamide and the third Danish group consisted of 413 middle-aged unrelated Type II diabetic patients who were consecutively recruited from the outpatient clinic at Steno Diabetes Centre and 280 age-matched, unrelated, and glucose tolerant Danish control subjects, traced in the Central Population Register, who had been examined by an OGTT. The Danish studies were approved by the ethics committee of Copenhagen. The Swedish cohort comprised 1192 Swedish Caucasian men who had been examined by an OGTT at the age of 70 years and who were diagnosed with Type II diabetes according to the National Diabetes Data Group criteria as described previously [4]. For this study DNA was available from 639 men with normal glucose tolerance. This study was approved by the human ethics committee of the Medical Faculty of Uppsala University. Before participating in the study, informed consent was obtained from all subjects for the experiments which were carried out in accordance with the principles of the Declaration of Helsinki.

SSCP-heteroduplex scanning and detection of nucleotide substitutions. We analysed three polymerase chain reaction (PCR) segments, covering a total of 612 basepairs upstream of the ATG start site (Table 1), by the SSCP-heteroduplex technique on genomic DNA extracted from leukocytes from 71 Type II diabetic patients. We also analysed five PCR amplified segments covering 14 tyrosine residues on genomic DNA from 318 Type II diabetic patients. The SSCP-heteroduplex formation analyses were carried out at two different experimental settings as described previously [5]. The nucleotide substitution at codon 647 was detected by a PCR amplified assay di- 
gesting with restriction enzyme Bst NI (New England Biolabs, Beverly, Mass., USA). The nucleotide substitution at codon 1057 was detected by digestion of a PCR product with either Srf I (Stratagene, La Jolla, Calif., USA) or Hha I (New England Biolabs).

Yeast two-hybrid assay. The interaction between the murine IRS-2 kinase regulatory loop binding (KRLB) domain and the insulin receptor and p85 of phosphatidylinositol (PI) 3-kinase, respectively, was estimated by cotransforming yeast cells with two-hybrid plasmids [6]. Briefly, the human insulin receptor cytoplasmic domain (amino acids 944-1343) was inserted in-frame with the DNA binding domain of LexA [6], the human $\mathrm{p} 85 \alpha$ subunit was inserted in-frame with the DNA binding domain of lexA of the pVJL-HIR vector (containing the repressible promoter MET25 in front of the insulin receptor cytoplasmic domain [7]), and the wildtype murine IRS-2 KRLB domain (amino acids 591-786) or the IRS-2 KRLB domain with a valine at codon 647 were both inserted in-frame with the Gal4 DNA activation domain. The basal interaction between the insulin receptor and the IRS-2 KRLB domain was determined by substituting the cDNA of the insulin receptor cytoplasmic domain with a cDNA construct mutated to eliminate kinase activity. The basal interaction with $\mathrm{p} 85 \alpha$ was determined by addition of $5 \mathrm{mmol} / \mathrm{l} \mathrm{L}$-methionine to the medium and plates which represses the expression of the insulin receptor $\beta$-subunit and thereby eliminates tyrosine phosphorylation.

The specific interaction between the insulin receptor and the wildtype or the mutated KRLB domain of IRS-2 was assessed by a fluid calorimetric $\beta$-galactosidase assay [6, 7].

The assay measuring the insulin receptor and IRS-2 KRLB interaction was carried out eight times in triplicate from two independent transformations, and the assay measuring the p $85 \alpha$ and IRS- 2 KRLB interaction was carried out 21 times in triplicate from two independent transformations.

Statistical analysis. The Statistical Package of Social Science (SPSS) for Windows was used for statistical analysis. A MannWhitney test was used for comparison between groups. A $p$-value $<0.05$ (two-tailed) was considered significant. Data in text and Table 2 are mean \pm SD. The data obtained by the yeast-two hybrid system were evaluated as mean $\pm \mathrm{SE}$, adjusted for protein expression level, and compared using a one-sample t test.

\section{Results}

We analysed 612 basepairs upstream of the ATG start site (Table 1) comprising the promoter sequence on DNA from 71 Danish Type II diabetic patients. No nucleotide changes were detected. Seven tyrosine residues placed in potential recognition motifs for the SH2 domains (YXXM or YMXM) in addition to seven other tyrosine residues were scanned for mutations by carrying out five PCR amplifications comprising the tyrosine residues on genomic DNA from 318 Type II diabetic patients. Only one nucleotide substitution was detected which predicted a conservative amino acid change of leucine to valine at codon 647. An association study showed that the variant was present in 3 of 413 diabetic patients and absent in 280 glucose tolerant control subjects.

The clinical onset of diabetes in the three carriers was at age 46, 66 and 57 years, respectively, and the
Number

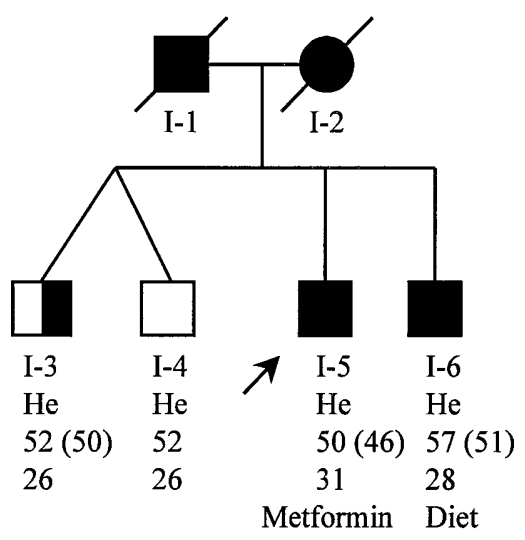

Number

Genotype

Age (age of diagnosis)

BMI

Treatment

Metformin Diet

Fig. 1. A Danish Caucasian pedigree with the Leu647Val IRS2 amino acid variant. Subjects with Type II diabetes are indicated by black symbols, subject with IGT by black and white symbol and subject with NGT by white symbol. The arrow indicates one of the three persons primarily defined as carrier of the amino acid variant. In the first line of the text below each subject is an identification code for each subject; line 2 indicates the genotype of the variant (He; heterozygous) (DNA was not available from subjects I-1 and I-2); line 3 indicates the current age and in parenthesis the age of onset of diabetes or IGT; line 4 indicates BMI in $\mathrm{kg} / \mathrm{m}^{2}$ of the subjects at the time of examination; line 5 describes the current treatment in case of diabetes (subject I-6 was initially treated by oral hypoglycaemic drugs but can manage on diet alone after a weight reduction of $8 \mathrm{~kg}$ )

treatment was metformin, sulphonylurea and insulin, respectively. Family data were accessible on one of the three carriers (Fig. 1), a man now aged 50 years (diabetes onset at age 46 years) with three older brothers of whom the middle brothers were dizygotic twins. The parents had died and no DNA was available, however, they had both been diagnosed with diabetes. Genotyping of the three brothers showed the presence of the Leu647Val variant. It is note worthy that the oldest brother was diabetic and one of the twins had impaired glucose tolerance. The other twin had a normal fasting plasma glucose concentration $(5.4 \mathrm{mmol} / \mathrm{l})$, and normal glucose concentration at $120 \mathrm{~min}$ during an OGTT $(5.9 \mathrm{mmol} / \mathrm{l})$, however, his $30 \mathrm{~min}$ and $60 \mathrm{~min}$ post-OGTT plasma glucose concentrations were increased. An estimation of the 30 and 60 min plasma glucose percentiles of 121 normal glucose tolerant middle-aged men showed that the 30 and 60 min glucose concentrations of the normal glucose tolerant twin were in the 90th percentile and 95th percentile, respectively, whereas the serum insulin concentrations did not differ.

The amino acid substitution at codon 647 is located in the KRLB (kinase regulatory loop binding) domain, which has previously been shown to interact with the insulin receptor. We expressed the wildtype and mutated murine IRS-2 KRLB domains, respectively, in the yeast two-hybrid system and examined the interaction with the human insulin receptor and 
human $p 85 \alpha$ to evaluate whether the IRS- 2 amino acid variant had any impact on these interactions. The $\beta$-galactosidase assay done in transformed yeast cells showed that the mean calculated binding between mutated or wildtype IRS-2 KRLB domain and the insulin receptor in the absence of phosphorylated tyrosine residues was similar (results not shown), whereas the binding of mutated IRS-2 KRLB to the insulin receptor in the presence of tyrosine phosphorylation was slightly but not significantly increased compared with the binding of wildtype IRS-2 KRLB to the insulin receptor [wildtype $=100 \%$ and Leu647Val $=119 \%$ (95\% confidence interval: 84-154)]. Similarly, there was no important difference between the interaction of mutated or wildtype IRS-2 KRLB domain and p85 $\alpha$ either in the absence (results not shown) or in the presence of tyrosine phosphorylation [wildtype $=100 \%$ and Leu647Val $=106 \%(101-112)]$.

To asses a potential impact on insulin secretion and insulin sensitivity of the previously identified IRS-2 glycine to aspartic acid polymorphism at codon 1057 , we examined four groups of subjects. The first group consisted of 363 young healthy Danish Caucasians who had the variant with an allelic frequency of $37.9 \%$ (95\% confidence interval: $33.0-42.8)$ and the second group consisted of 228 Danish Caucasian glucose-tolerant offspring of one Type II diabetic parent. The frequency in this group was $34.2 \%$ (29.7-38.3). The allelic frequency was previously determined to be $33.9 \%(29.6-38.2)$ in a third group of 236 middle-aged Danish Caucasians [2]. In the last cohort comprising 639 Swedish glucose tolerant men, the allelic frequency was $34.5 \%(30.8-38.2)$.

The young healthy subjects and the glucose-tolerant offspring carrying the homozygous IRS-2 variant showed no detectable differences in their beta-cell function during an IVGTT compared with wildtype subjects (Table 2). The fasting and $30 \mathrm{~min}$ post-glucose serum insulin and C-peptide concentrations of the glucose tolerant offspring were analysed applying a variance component model which allowed an extra source of variation to account for a possible correlation between subjects of the same family. There were still no differences found between carriers of the variant and wildtype carriers (data not shown). In contrast, examination of the middle-aged Danish glucose tolerant subjects (Table 2) showed that the fasting serum insulin concentrations of homozygous carriers were decreased by $25 \%$ compared with wildtype carriers $(p=0.009)$ and the serum C-peptide concentrations were decreased by $17 \%(p=0.001)$. Despite the reductions in fasting serum insulin and C-peptide concentrations there were no concomitant alterations in the fasting plasma glucose concentrations of the carriers of the homozygous polymorphism. The serum insulin and C-peptide concentrations of the homozygous subjects remained signifi- cantly decreased during the OGTT compared with wildtype carriers (Table 2). After adjusting for sex and BMI in a multivariate analysis, the reductions in serum insulin concentrations of homozygous carriers remained significant at 30 and $60 \mathrm{~min}$ during the OGTT, likewise, the reductions in C-peptide concentrations remained significant at 30 and 60 min during the OGTT as well as during fasting.

In an attempt to replicate the findings in the middle-aged Danes we examined the cohort of 639 glucose tolerant Swedish men aged 70 years (Table 2). In this cohort no differences in fasting or postOGTT plasma insulin concentrations were detected between wildtype and homozygous carriers.

The whole body insulin sensitivity was measured with a tolbutamide-modified IVGTT in both the young healthy subjects and in the glucose-tolerant first degree relatives of a Type II diabetic patient. The codon 1057 polymorphism had no significant effect on the insulin sensivity index (Table 2). When subjects in the four groups were stratified in accordance to BMI below or equal to and above $25 \mathrm{~kg} / \mathrm{m}^{2}$, there was no evidence for an interaction between obesity and the codon 1057 variant with respect to insulin secretion or insulin sensitivity (data not shown).

We have previously reported about a lean healthy Danish man (23 years) who was homozygous for a glycine to arginine amino acid variant at codon 972 in IRS-1 $[5,8,9]$. Due to his low concentrations of fasting serum insulin and $\mathrm{C}$ peptide we determined the IRS-2 genotype and disclosed that the person was homozygous for the IRS-2 variant as well. The fasting serum insulin and C-peptide percentiles of 50 young and lean men carrying both IRS- 1 and -2 wildtype proteins were evaluated. This analysis showed that serum insulin and C-peptide concentrations of the double homozygous carrier were placed in the lower fifth percentile of the reference group. The double homozygous carrier and five male wildtype IRS-1 carriers were challenged by a dexamethasone suppression test and by the end of the test, the homozygous carrier showed an overt but transient diabetes compared with the five wildtype carriers [8].

\section{Discussion}

Genetic variability of the promoter sequence potentially causes alterations in the levels of protein expression, however, our study showed no changes in the putative promoter sequence of IRS-2 in diabetic patients, suggesting that variations are rare at least in Scandinavians.

Scanning of genomic DNA surrounding 14 tyrosine residues, of which seven tyrosines were located within motifs recognized by the $\mathrm{SH} 2$ domains, disclosed a novel variant at codon 647 present in Type II diabetic patients only. Analysis of the family of 
Table 2. Biochemical and clinical characteristics obtained during an IVGTT in combination with intravenous injection of tolbutamide from 363 young healthy Danish Caucasians and 228 Danish glucose tolerant offspring of one Type II diabetic parent and data obtained during an OGTT in 236 middle-aged glucose tolerant Danes and 639 Swedish glucose tolerant males aged 70 years carrying either the wildtype, the heterozygous or the homozygous Gly1057Asp polymorphism in IRS-2

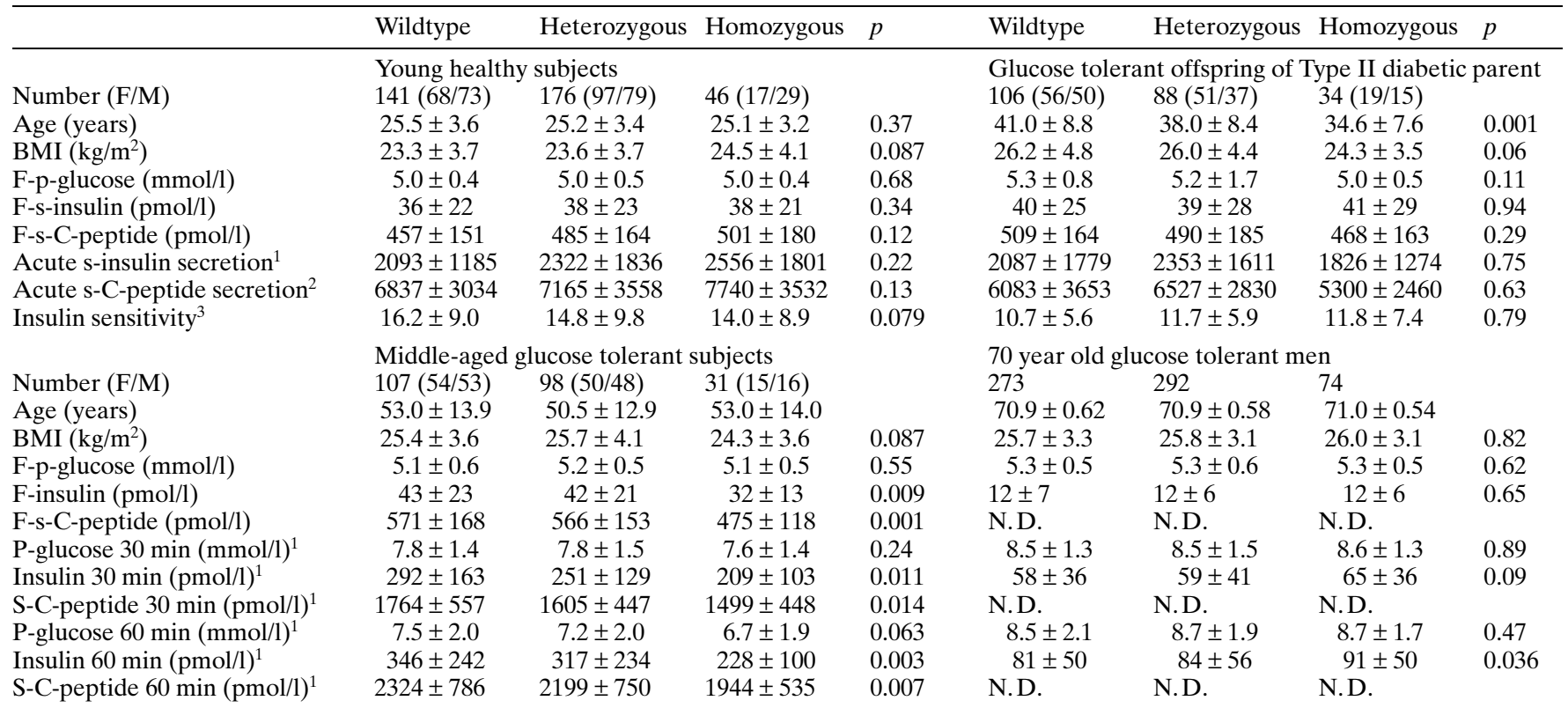

Values are means \pm SD. The $p$ values obtained by the MannWhitney test compare subjects homozygous for the codon 1057 polymorphism with wildtype carriers. F denotes fasting, $\mathrm{p}$ denotes plasma, s denotes serum, N.D. is not determined, ${ }^{1}$ denotes post-OGTT. ${ }^{2}$ Area under curve $0-8 \mathrm{~min}(\mathrm{pmol} / \mathrm{l})$ after IVGTT. ${ }^{3}$ Insulin sensitivity index estimated by Bergman's minimal model $\left(\mathrm{x} 10^{-5} \mathrm{lmin}^{-1} \mathrm{pmol}^{-1}\right)$ after intravenous injection of $0.3 \mathrm{~g}$ glucose per $\mathrm{kg}$ body weight over $1 \mathrm{~min}$ followed 20 mins later by injection of a bolus of $3 \mathrm{mg}$ tolbutamide per kg body weight [8]. The insulin concentrations during the OGTT were measured with different assays in Danish and
Swedish subjects. Statistical analyses of serum insulin and Cpeptide concentrations are evaluated as logarithmic values.

In the studies of Danes, plasma glucose was analyzed by a glucose oxidase method whereas plasma glucose in Swedish subjects was measured by a glucose dehydrogenease method. Serum specific insulin (excluding des $(31,32)$ ) was measured in Danes by ELISA and plasma insulin in Swedish subjects was analysed using an enzymatic-immunological assay. Serum C-peptide was determined by radioimmunoassay using Steno Diabetes Centre routine methods. one of the carriers showed diabetes in the parents and in one brother (Fig. 1) and impaired glucose tolerance in another brother. The last brother had normal fasting and $120 \mathrm{~min}$ post-glucose load plasma glucose concentrations although his 30 and 60 min glucose concentrations were increased. The observed increases in plasma glucose concentrations represent two non-standard time points and the significance of this observation is not clear. Taken together, these results of the carriers of the variant in codon 647 do not allow to state whether the polymorphism possibly contributes to rare cases of diabetes or its association with diabetes is a chance occurrence.

The amino acid substitution at codon 647 is located in the KRLB domain, a region between amino acids 591-786, which in addition to the pleckstrin homology domain and the phosphotyrosine binding domain binds to the insulin receptor [6]. Two tyrosine residues in the murine KRLB domain located at position 624 and 628 , corresponding to tyrosine 628 and 632 in human IRS-2, respectively, have been shown to be important in mediating the insulin receptor binding [6]. In addition, tyrosine residue 653 in human IRS-2 is residing in a YMXM motif which in the corresponding position of human IRS-1 is a binding site for $\mathrm{p} 85 \alpha$, the regulatory subunit of phosphatidylinositol (PI) 3-kinase. Therefore, we expressed the wildtype and mutated murine IRS-2 KRLB domains, respectively, in the yeast two-hybrid system [the human IRS-2 cDNA was not available, nevertheless, the KRLB domains are highly conserved among species (6)] and examined the interaction with the human insulin receptor and human $\mathrm{p} 85 \alpha$, to evaluate whether the IRS-2 amino acid variant had any impact on these interactions. In this simplified system, however, there were no differences due to the amino acid substitution in the binding to the insulin receptor or $\mathrm{p} 85 \alpha$.

Recent results have shown that secreted insulin acts by way of insulin receptors on pancreatic betacell and thereby up-regulates insulin gene transcription by signalling through the IRS-2/PI 3-kinase/p70 S6kinase pathway [10]. Accordingly, we determined whether the common IRS-2 glycine to aspartic acid 
polymorphism at codon 1057 had any impact on insulin secretion and furthermore on insulin sensitivity in four different population groups. The conflicting results obtained on insulin secretion between young and middle aged Danish glucose-tolerant population groups might indicate that the potential effect of the IRS-2 variant is not detectable in early adult life but develops as a result of age-related changes in insulin secretion and insulin action. Thus, this hypothesis was tested indirectly in an attempt to replicate the findings in the middle-aged Danes by examination of glucose tolerant Swedish men aged 70 years (Table 2). Nevertheless, in this elderly population the amino acid variant seems to play no part in insulin secretion. Finally, the amino acid variant had no effect on insulin sensitivity in the examined populations

In conclusion: we did not discover any genetic variation in the IRS-2 promoter. A conservative polymorphism in IRS-2 was identified in the KRLB-domain at codon 647 . The variant has so far only been identified in rare cases of Type II diabetic patients. The amino acid change did not affect the interaction between the IRS-2 KRLB domain and the insulin receptor and p85 $\alpha$ of PI 3-kinase, respectively, as measured in the yeast two-hybrid system. Data presented here indicate that the prevalent variant of IRS-2 at codon 1057 has no consistent effect on glucose-induced insulin secretion or insulin sensitivity in glucose tolerant subjects.

Acknowledgements. This study was supported by grants from the University of Copenhagen, the Danish Medical Research Council, the Danish Diabetes Association, the Velux Foundation, EEC (BMH4-CT-950662) and the American Diabetes Association and National Institutes of Health (NIH) Grant DK-43808 to M.F. White and D. Bernal was supported by a FPI Fellowship from the Spanish Government. The authors wish to thank A. Forman, B. Mottlau, L. Aabo, S. Urioste, L. Drastrup, D. Gøth-Johansen, S. Kjellberg and J. Brønnum for technical assistance and G. Lademann for secretarial assistance.

\section{References}

1. Withers DJ, Gutierrez JS, Towery H et al. (1998) Disruption of IRS-2 causes type-2 diabetes in mice. Nature 391: 900-904

2. Bernal D, Almind K, Yenush L et al. (1998) IRS-2 amino acid polymorphisms are not associated with random type 2 diabetes among Caucasians. Diabetes 47: 976-979

3. Clausen JO, Borch-Johnsen K, Ibsen H et al. (1996) Insulin sensivity index, acute insulin-response and glucose effectiveness in a population-based sample of 380 young healthy Caucasians- Analysis of the impact of gender, body-fat, physical fitness and life-style factors. J Clin Invest 98: 1195-1209

4. Hedstrand H (1975) A study of middle-aged men with particular reference to risk factors for cardiovascular diseases. Uppsala J Med Sci 80: 1-61

5. Almind K, Bjørbæk C, Vestergaard H, Hansen T, Echwald S, Pedersen O (1993) Amino-acid polymorphisms of insulin receptor substrate-1 in non-insulin- dependent diabetes mellitus. Lancet 342: 828-832

6. Sawka-Verhelle D, Baron V, Mothe I, Filloux C, White MF, Van Obberghen E (1997) Tyr (624) and Tyr (628) in insulin receptor substrate-2 mediate its association with the insulin-receptor. J Biol Chem 272: 16414-16420

7. Delahaye, L, Mothe-Satney I, Myers MG, White MF, Van Obberghen E (1998) Interaction of insulin receptor substrate-1 (IRS-1) with phosphatidylinositol 3-kinase:Effect of substitution of serine for alanine in potential IRS-1 serine phosphorylation sites. Endocrinology 139: 4911-4919

8. Clausen JO, Hansen T, Bjørbæk C et al. (1995) Insulin-resistance - interactions between obesity and a common variant of insulin-receptor substrate-1. Lancet 346: 397-402

9. Almind K, Inoue G, Pedersen O, Kahn CR (1996) A common amino-acid polymorphism in insulin-receptor substrate- 1 causes impaired insulin signaling - evidence from transfection studies. J Clin Invest 97: 2569-2575

10. Leibiger IB, Leibiger B, Moede T, Berggren P (1998) Exocytosis of insulin promotes insulin gene transcription via the insulin receptor/PI-3 kinase/p70 s6 kinase and CaM kinase pathways. Mol Cell 1: 933-938 